\title{
Teneurs en métaux traces des eaux et poissons de cinq étangs de Dakar et risques pour la santé humaine
}

\author{
DIOP Cheikh ${ }^{1 *}$, DIATTA Arthur1, ${ }^{2}$, NDIAYE Astou², CABRAL Mathilde1, TOURE Aminata ${ }^{1}$, FALL \\ Mamadou1 \\ 1: Laboratoire de Toxicologie et Hydrologie, Université Cheikh Anta DIOP de Dakar BP 5005 Dakar Fann (Sénégal). \\ 2: Laboratoire National d'Analyse et de Contrôle, Ministère du Commerce du Sénégal, Dakar (Sénégal). \\ *Auteur pour la correspondance : Cheikh DIOP : Laboratoire de Toxicologie et Hydrologie, Faculté de Médecine, de \\ Pharmacie et d'Odontologie, Université Cheikh Anta DIOP de Dakar BP 5005 Dakar Fann, Sénégal. \\ Email: cheikh4.diop@ucad.edu.sn / cheikhkoki@hotmail.com Tel: (221) 338246539
}

Original submitted in on $13^{\text {th }}$ February 2019. Published online at www.m.elewa.org/journals/ on $31^{\text {st }}$ May 2019 https://dx.doi.org/10.4314/jab.v137i1.1

\section{RESUME}

Objectifs : Les objectifs de cette étude étaient de déterminer le niveau de concentration en métaux traces (Cadmium, Plomb, Fer) de la colonne d'eau et du tilapia (poisson la plus consommée au Sénégal) échantillonnés sur des étangs de Dakar afin d'évaluer les risques potentiels pour la santé humaine liés à leur consommation.

Méthodologie et Résultats: Les échantillons d'eau et de poisson ont été collectés dans 5 étangs (Mbeubeuss, Mbao, Technopôle, Cambérène et Parc Hann) et les variations des concentrations en métaux traces entre sites et organes ont été étudiées après dosage par spectrométrie d'absorption atomique. Seules les concentrations de $C d$ dans l'eau à l'étang de Mbeubeuss $\left(0,22 \mu \mathrm{g} . \mathrm{I}^{-1}\right)$ étaient supérieures aux critères sur la protection de l'eau $\left(0,2 \mu \mathrm{g} . \mathrm{I}^{-1}\right)$. Les teneurs en $\mathrm{Pb}$ et en $\mathrm{Cd}$ dans la chair des tilapias étaient inférieures aux normes recommandées par l'Union Européenne et ne constituent pas un risque pour la santé humaine.

Conclusions et applications: Le foie des poissons a enregistré les teneurs les plus élevées en métaux traces en tout site. Le calcul du quotient de danger pour le $\mathrm{Pb}$ et le $\mathrm{Cd}$ a révélé qu'il n'y avait pas de risques sanitaires liés à la consommation des tilapias pêchés dans ces étangs. Ces résultats pourraient servir aux autorités publiques pour recommander la consommation des poissons tout en renforçant la préservation des zones humides dans le cadre d'un développement durable.

Mots clés : Etangs, Colonne d'eau, Tilapia, Métaux traces, Risque sanitaire

Concentrations and human health risks of trace metals in water and tilapia from five ponds of Dakar

\section{ABSTRACT}

Objectives: The objectives of this study were to determine the concentration of trace metals (Cadmium, Lead and Iron) of the water column and tilapia (fish most consumed in Senegal) sampled on Dakar ponds in order to evaluate the potential risks to human health related to their consumption. 
Methodology and Results: The water and fish samples were collected in 5 ponds (Mbeubeuss, Mbao, Technopole, Cambérène and Parc Hann) and the variations in trace metals concentrations between sites and organs were investigated after atomic absorption spectrometric determination. Only $\mathrm{Cd}$ concentrations in water of Mbeubeuss pond $\left(0.22 \mu \mathrm{g} . \mathrm{I}^{-1}\right)$ exceeded the water protection criteria $\left(0.2 \mu \mathrm{g} . \mathrm{I}^{-1}\right)$. The results showed that concentrations of $\mathrm{Cd}$ and $\mathrm{Pb}$ in tilapia meat were below the limit values established by the European Community and pose no threat to public health

Conclusions and application of findings: The fish liver recorded the highest levels of trace metals at any site. The calculation of the hazard quotient for $\mathrm{Pb}$ and $\mathrm{Cd}$ revealed that there was no health risks related to the consumption of tilapia caught in these ponds. These results could be used by public authorities to recommend fish consumption while strengthening the preservation of wetlands as part of sustainable development.

Keywords: Ponds, Water column, Tilapia, Trace metals, Health risk

\section{INTRODUCTION}

Dans le monde, de nombreuses zones humides souffrent de pollution, affectant de manière significative les écosystèmes aquatiques et les pêcheries. Au cours des dernières décennies, de nombreuses études ont porté sur le niveau de contamination du milieu aquatique et un intérêt croissant pour la contamination des produits halieutiques consommables en relation avec les risques pour la santé (Storelli, 2008; Bandowe et al, 2014, Amara et al, 2019). La consommation des produits halieutiques, et plus précisément celle de poissons de par leur qualité nutritionnelle et leurs effets bénéfiques pour la santé, est en nette progression à travers le monde. En effet, l'offre mondiale de poissons est passée de 9,8 $\mathrm{kg} /$ personne/an dans les années 1960 à 20 $\mathrm{kg} /$ personne/an en 2016 (FAO, 2016). Cependant, la consommation de poissons peut être une source importante d'exposition à de nombreux contaminants chimiques présents dans l'environnement (Storelli, 2008). De nombreuses études ont montré que la contamination par les métaux traces (cadmium, plomb) de la chaîne alimentaire pouvait présenter un risque pour la santé humaine (Taweel et al, 2013; lqbal and Shah, 2014). Pour limiter l'impact potentiel de ces polluants sur la santé humaine, plusieurs agences et organisations telles que I'Union européenne (UE) ont établi des limites maximales pour le $\mathrm{Cd}$ et le $\mathrm{Pb}$ dans le poisson. II est donc important d'évaluer les risques pour la santé liés à la consommation de poissons potentiellement contaminés par ces métaux traces via le régime alimentaire. Au Sénégal, pays en développement, les produits halieutiques sont la principale source de protéines animales. Le poisson et les fruits de mer constituent $75 \%$ de la source de protéines de la population (ANSD, 2009). En effet, les eaux intérieures du Sénégal abritent d'importantes ressources halieutiques dont une partie est exportée vers les marchés africain, américain, asiatique et européen, le reste est consommé localement (Amara et al, 2019). Cependant, l'urbanisation rapide et incontrôlée de la région de Dakar a entrainé de graves problèmes environnementaux tels que l'augmentation de la pollution des zones humides de la région (ANSD, 2013). Ces zones qui abritent les étangs où sont péchés des pissons subissent une forte agression anthropique liée aux importantes activités tels que le maraîchage, la pêche, le pâturage, l'élevage qui s'y développent. Par conséquent, elles deviennent un dépotoir de déchets générés par l'homme pouvant impacter négativement les poissons et en conséquence la santé des consommateurs. Les objectifs de la présente étude étaient de déterminer le niveau de concentration en métaux traces $(\mathrm{Cd}, \mathrm{Pb}, \mathrm{Fe})$ de la colonne d'eau et du tilapia (espèce de poisson la plus consommée au Sénégal) échantillonnés sur cinq étangs de Dakar, d'étudier la variation de la concentration de ces contaminants entre organes (chair et foie) et entre sites et d'évaluer les risques potentiels pour la santé humaine liés à leur consommation. 


\section{MATERIEL ET METHODES}

Zone d'étude et échantillonnage: Cinq points de prélèvement de l'eau sont sélectionnés de manière aléatoire dans chacun des cinq sites: Mbao, Technopôle, Mbeubeuss, Cambérène et Parc Hann (Figure 1). Le prélèvement des échantillons d'eau $(n=$ 25) s'est effectué selon le protocole d'échantillonnage de métaux à l'état de traces dans les eaux de surface (MDDELCC, 2014). II a été réalisé à dix mètres de la rive à l'aide de bouteilles neuves préalablement décontaminées, traitées et ensachées la veille. Sur le site de prélèvement, l'eau est prélevée dans des flacons en polyéthylène de $250 \mathrm{ml}$ contenant $0,50 \mathrm{ml}$ d'acide nitrique concentré, à titre d'agent de conservation jusqu'à son épaulement. Les flacons sont ensuite fermés hermétiquement et transportés au laboratoire à $4{ }^{\circ} \mathrm{C}$. En ce qui concerne la collecte des échantillons de poissons, elle s'est faite en collaboration avec les pêcheurs retrouvés dans les différents sites de l'étude. Ainsi, cinq tilapias ont été échantillonnés par site. Des individus de même taille ont été prélevés sur chaque étang pour s'affranchir de la variabilité de la concentration des contaminants en fonction de la taille. Au total, 20 échantillons de tilapias (longueur total de 16,33 $\pm 1,89 \mathrm{~cm}$ ) ont été prélevés en fin août 2016 puis congelés à $-20^{\circ} \mathrm{C}$ jusqu'à ce que le dosage des métaux traces puisse être effectué.

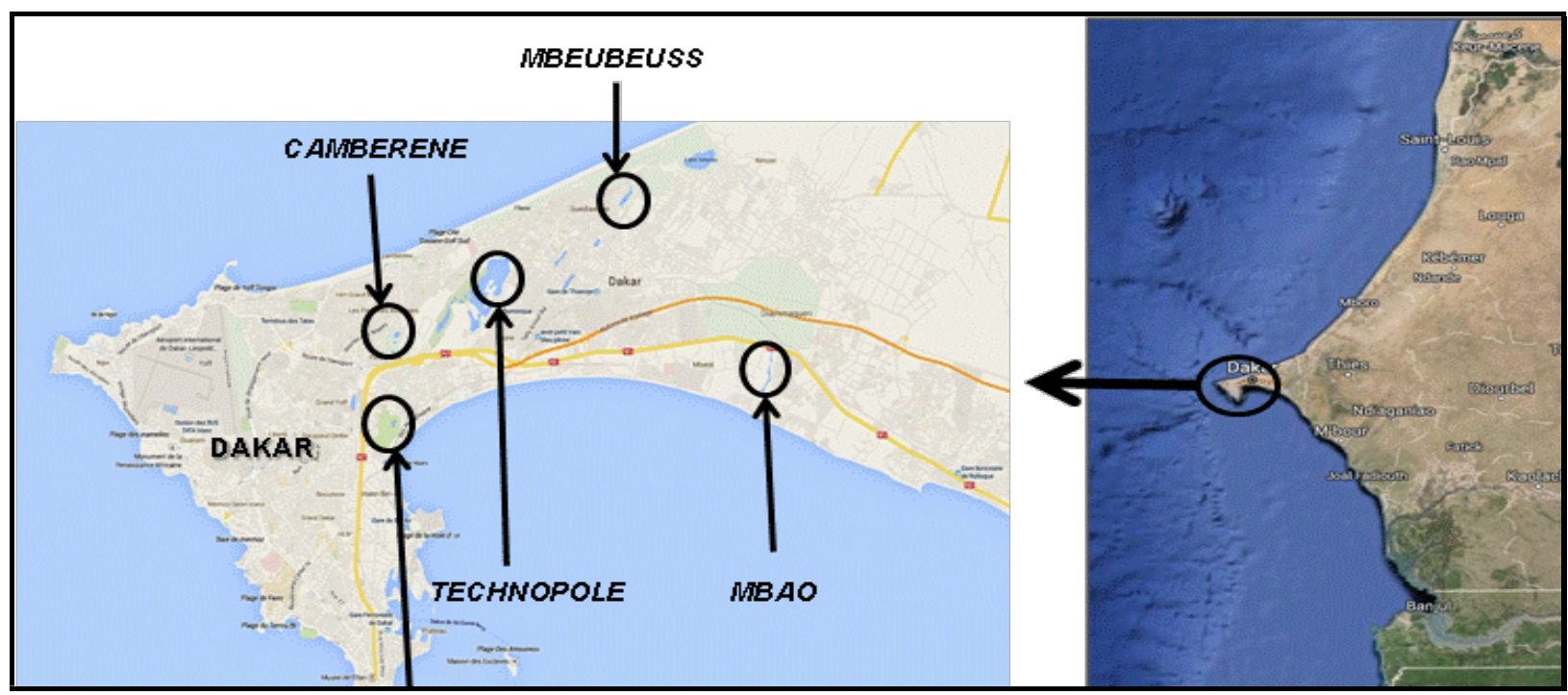

Figure 1: Carte des sites d'échantillonnage

Préparation des échantillons d'eaux et de poissons : Toute la verrerie de laboratoire est nettoyée avec un détergent et de l'eau de robinet puis rincée vigoureusement avec de l'eau bi distillée avant utilisation. Pour la préparation des échantillons d'eau bien homogénéisés, $30 \mathrm{ml}$ d'eau sont prélevés et déposés dans une bombe en téflon. De l'eau régale (acide chlorhydrique et acide nitrique : $3 / 1: \mathrm{v} / \mathrm{v}$ ) y a été ajoutée. Ce mélange est laissé sous hotte pendant 10 minutes avant d'être introduit le four à micro-ondes pour minéralisation. S'agissant de la préparation des échantillons de poissons, ils ont été décongelés puis écaillés et les viscères vidés. Sur chaque poisson, deux parties sont prélevées : la chair prise au niveau du pédoncule caudal et le foie. Dans les bombes en téflon, grâce à une balance de précision de marque Radwag vérifiée et étalonnée, une prise d'essai d'environ $0,5 \mathrm{~g}$ de chair et 0,15 à $0,5 \mathrm{~g}$ de foie y est déposée puis 10 $\mathrm{ml}$ d'acide nitrique $65 \%$ y ont été ajoutés. Les bombes en téflon sont ensuite laissées sous la hotte pendant 20 minutes avant de les fermer hermétiquement et de les placer dans le four à micro-ondes TOP wave Analytik Jena AG pour minéralisation. Celle-ci est faite suivant le programme de minéralisation donné par le fabricant (application poisson 42 pour le foie et la chair et programme water Epa pour les échantillons d'eau). A la fin de la minéralisation et après refroidissement, les bombes en téflon sont retirées du four et le minéralisât est récupéré sous hotte dans des fioles de $100 \mathrm{ml}$ avec de l'eau milli $Q$ pour analyse. Pour vérifier qu'il n'y a pas eu de contamination, un essai à blanc est inclus dans chaque série d'analyse dans les mêmes conditions que les échantillons d'eau.

Dosage par Spectromètre d'Absorption Atomique: Le dosage du $\mathrm{Pb}$, du $\mathrm{Cd}$ et de Fe dans l'eau et les poissons tilapias est effectué à l'aide d'une 
spectrométrie d'absorption atomique (VARIAN SPECTRAA 220FS) avec une limite de quantification de $0,1 \mathrm{mg} \cdot \mathrm{kg}^{-1}$ pour le $\mathrm{Cd}$ et le $\mathrm{Pb}$. Les courbes d'étalonnage ont été établies à partir des solutions standards de $\mathrm{Pb}, \mathrm{Cd}$ et de $\mathrm{Fe}$ à $1000 \mathrm{ppm}$ de métal dissous dans de l'acide nitrique $63 \%$ et d'eau déminéralisée.

\section{Traitement des données et analyse statistique}

Traitement des données : Les concentrations (C) en

$\mathrm{Pb}, \mathrm{Cd}$ et Fe sont exprimées en mg.kg ${ }^{-1}$ de poids frais selon la formule suivante :

$$
\mathrm{C}\left(\mathrm{mg} \cdot \mathrm{kg}^{-1}\right)=\frac{(\mathrm{Cs}-\mathrm{Cb}) \times \mathrm{Vf}}{\text { PEx1000 }} \text { avec }
$$

C: concentration finale de l'élément en $\mathrm{mg}^{\mathrm{kg}}{ }^{-1}$;

Cs: concentration en $\mu g . I^{-1}$ de l'élément dans

l'échantillon ; $\mathrm{Cb}$ : concentration en $\mu \mathrm{g} . \mathrm{l}^{-1}$ de l'élément dans le blanc; Vf : volume final $(100 \mathrm{ml})$; PE : prise d'essai en gramme (poids frais) de l'échantillon.

Le calcul du facteur de bioconcentration $\left(\mathrm{FBC}=\frac{\mathrm{Cm}}{\mathrm{Ce}}\right)$ est inspirée de la formule de Casas (2005) avec :

$\mathrm{Cm}$ : concentration en métaux dans le poisson et

$\mathrm{Ce}$ : concentration en métaux dans l'eau

La comparaison de la teneur totale en métaux entre les différents sites d'échantillonnage a été réalisée à partir du calcul de l'indice de pollution des métaux (Khillare et al, 2012):

IPM $=\left(C_{C d} \times C_{P b}\right)^{1 / 2}$ où $C_{C d}$ et $C_{P b}$ sont respectivement les concentrations de $\mathrm{Cd}$ et de $\mathrm{Pb}$ dans l'échantillon. $\mathrm{La}$ Dose Journalière d'Exposition (DJE) et le quotient de danger (THQ) sont calculés pour évaluer le risque pour

\section{RESULTATS}

Distribution des métaux dans l'eau: Les concentrations moyennes du $\mathrm{Pb}$, du $\mathrm{Cd}$ et du Fe dans l'eau des cinq sites étudiés sont consignées dans le tableau 1. Ce tableau montre que les eaux des étangs sont plus concentrées en plomb qu'en cadmium et que la santé. La DJE est estimée en fonction de la concentration maximale des métaux et du poids corporel. Son expression analytique $(\mathrm{mg} / \mathrm{kg} / \mathrm{j})$ est la suivante : $\mathrm{DJE}=\frac{\mathrm{CO} \times \mathrm{C}}{\mathrm{PC}}$ où

CO correspond à la consommation de poisson : 0,043 $\mathrm{kg} / \mathrm{j})(\mathrm{FAO}, 2014)$; $\mathrm{C}$ correspond à la concentration du métal dans le poisson et PC correspond au poids corporel moyen $(60 \mathrm{~kg})$.

Le THQ correspond au ratio entre l'exposition à un contaminant et l'exposition considérée sans risque (USEPA, 2000 ; Storelli, 2008 ; Bandowe et al, 2014 ; Diop et al, 2017). II est décrit par la formule simplifiée suivante : $T H Q=\frac{\text { DJE }}{\mathrm{D} J \mathrm{~T}}$ où

THQ correspond au quotient de danger (Target Hazard Quotient), DJE est la dose journalière d'exposition en $\mathrm{mg} / \mathrm{kg} / \mathrm{j}$ et DJT correspond à la valeur toxicologique de référence $\left(2.10^{-3} \mathrm{mg} / \mathrm{kg} / \mathrm{j}\right.$ pour le $\mathrm{Cd}$ et $3,5 \cdot 10^{-3} \mathrm{mg} / \mathrm{kg} / \mathrm{j}$ pour le $\mathrm{Pb}$ ) (Bisson et al, 2009).

Analyse statistique: L'analyse des résultats a été réalisée grâce au logiciel SPSS version 22 à l'aide duquel nous avons effectué l'analyse de la Variance (ANOVA). Le test de comparaison $\mathrm{T}$ de Student de deux échantillons indépendants nous a permis de montrer le niveau de différence entre les métaux traces selon les sites et organes. La procédure de Dunn/ Test bilatéral qui est une comparaison multiple par pair a été utilisée grâce au logiciel XLSTAT 2016 pour matérialiser par des lettres $(a, b, c)$ le niveau de significativité du P-value fixé à $5 \%$ au niveau des graphiques.

l'ordre des teneurs moyennes des métaux dans les échantillons des eaux de tous les sites se présente comme suit: $\mathrm{Fe}>\mathrm{Pb}>\mathrm{Cd}$. Dans certains sites comme Parc Hann et Technopôle, le Cd n'a pas été détecté.

Tableau 1: Concentrations moyennes ( \pm écart-type) des eaux en métaux par site $\left(\mu g . \mathrm{L}^{-1}\right)$

\begin{tabular}{l|c|c|c}
\hline Sites & $\mathbf{P b}$ & $\mathbf{C d}$ & Fe \\
\hline CAMBERENE & $1,05 \pm 0,35$ & $0,06 \pm 0,05$ & $0,54 \pm 0,84$ \\
\hline MBAO & $0,82 \pm 0,14$ & $0,08 \pm 0,01$ & $1,30 \pm 0,65$ \\
\hline MBEUBEUSS & $1,34 \pm 0,28$ & $0,22 \pm 0,06$ & $1,42 \pm 0,38$ \\
\hline PARC HANN & nd & nd & $4,00 \pm 0,34$ \\
\hline TECHNOPOLE & $0,07 \pm 0,16$ & nd & nd \\
\hline
\end{tabular}

nd: non détecté 
Par ailleurs, une différence statistiquement significative entre les concentrations de $\mathrm{Pb}$ à Mbeubeuss $(1,34$ $\left.\mu g . L^{-1}\right)$ et à Technopôle $\left(0,07 \mu \mathrm{g} \cdot \mathrm{L}^{-1}\right)$ a été notée de même qu'entre Cambérène $\left(1,05 \mu \mathrm{g} . \mathrm{L}^{-1}\right)$ et Technopôle $\left(0,07 \mu \mathrm{g} \cdot \mathrm{L}^{-1}\right)$. A l'exception du Fe dont la teneur au niveau du Parc Hann est trois fois supérieure à celle retrouvée dans les autres sites, l'étang de Mbeubeuss a enregistré les teneurs les plus élevées en $\mathrm{Pb}$ et en Cd dans l'eau.

Distribution des métaux dans le foie et la chair du tilapia: Les teneurs moyennes en métaux dans la chair et le foie des tilapias collectés dans les différents sites sont consignées dans le tableau 2.

Tableau 2:Teneurs moyennes ( \pm écart type) en métaux $\left(\mu \mathrm{g} . \mathrm{kg}^{-1}\right)$ des organes du tilapia

\begin{tabular}{l|l|l|l|l|l|l}
\hline \multirow{2}{*}{ Sites } & \multicolumn{4}{|l|}{ Chair } & Foie & \\
\cline { 2 - 7 } & $\mathbf{P b}$ & Cd & Fe & Pb & Cd & Fe \\
\hline CAMBERENE & $5,8 \pm 11,5$ & nd & $44,5 \pm 46,4$ & $46,1 \pm 32,9$ & $3,9 \pm 4,5$ & $350,1 \pm 126,4$ \\
\hline MBAO & $6,8 \pm 7,3$ & $2,3 \pm 2,3$ & $115,9 \pm 259,3$ & $135,8 \pm 71,1$ & $0,6 \pm 1,1$ & $2127 \pm 1936$ \\
\hline PARC HANN & $36,8 \pm 6,7$ & $3,3 \pm 1,3$ & $117,2 \pm 262,0$ & $144,8 \pm 60,8$ & $0,8 \pm 1,2$ & $3308 \pm 2859$ \\
\hline TECHNOPOLE & $20,7 \pm 23,6$ & $2,3 \pm 1,5$ & $31,9 \pm 11,3$ & $35,5 \pm 23,8$ & $1,9 \pm 2,4$ & $71,9 \pm 54,5$ \\
\hline
\end{tabular}

nd : non détecté

II apparait dans le tableau 2 que les plus grandes teneurs en $\mathrm{Pb}$ dans le foie et la chair du tilapia respectivement de $144,8 \mu \mathrm{g} . \mathrm{kg}^{-1}$ et $36,8 \mu \mathrm{g} \cdot \mathrm{kg}^{-1}$ sont observées dans le site Parc Hann. Toutefois, ces valeurs sont nettement inférieures à la norme européenne qui est de $300 \mu \mathrm{g} \cdot \mathrm{kg}^{-1}$ (EC, 2015). Ce même site enregistre les teneurs les plus élevées en $\mathrm{Cd}$ dans la chair alors que celles-ci sont plus élevées dans le foie des poissons du site de Cambérène. Dans tous les sites, les teneurs en $\mathrm{Cd}$ de la chair du poisson Tilapia sont inférieures à la norme requise par l'Union
Européenne qui est de $50 \mu \mathrm{g} \cdot \mathrm{kg}^{-1}$ (EC, 2014). La comparaison des différentes teneurs en $\mathrm{Pb}$ dans le foie montre des différences statistiquement significatives entre les sites Technopole et Mbao, Technopôle et Parc Hann et enfin Cambérène et Parc Hann (Figure 2) alors que dans la chair, seuls les sites Cambéreène et Parc Hann présentent ces différences (Figure 3). Dans tous les cas, l'ordre de grandeur des métaux dans la chair et le foie du Tilapia pour les sites est identique à celui retrouvé pour l'eau.

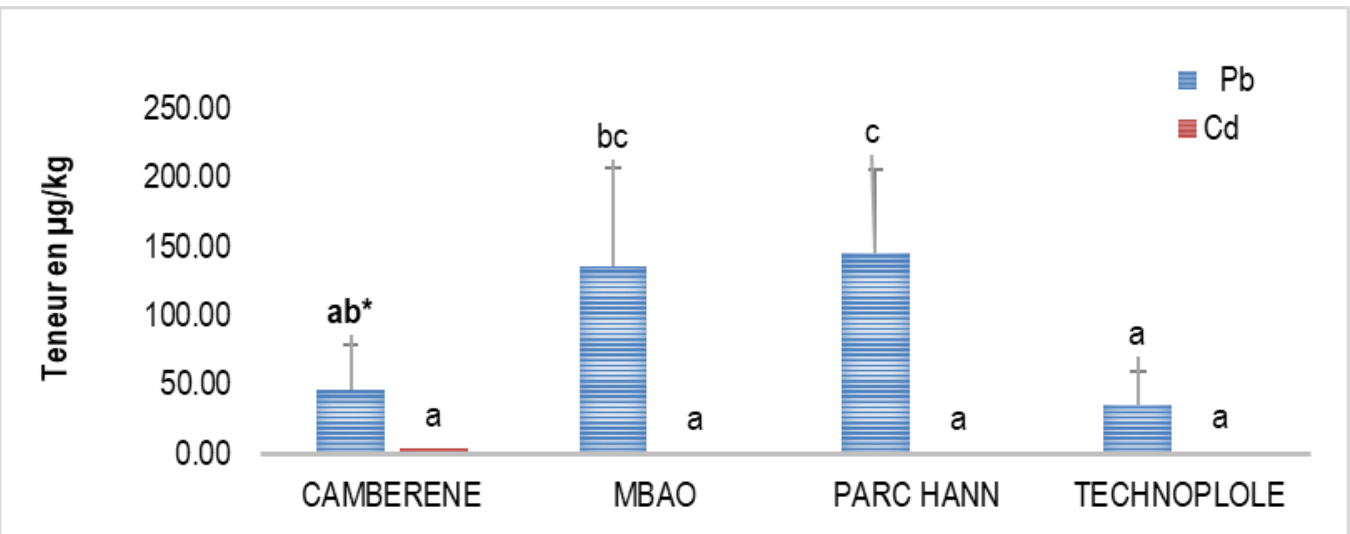

Figure 2: Distribution du $\mathrm{Pb}, \mathrm{Cd}$ et Fe dans le foie de Sarotherodon melanotheron $s p$ 


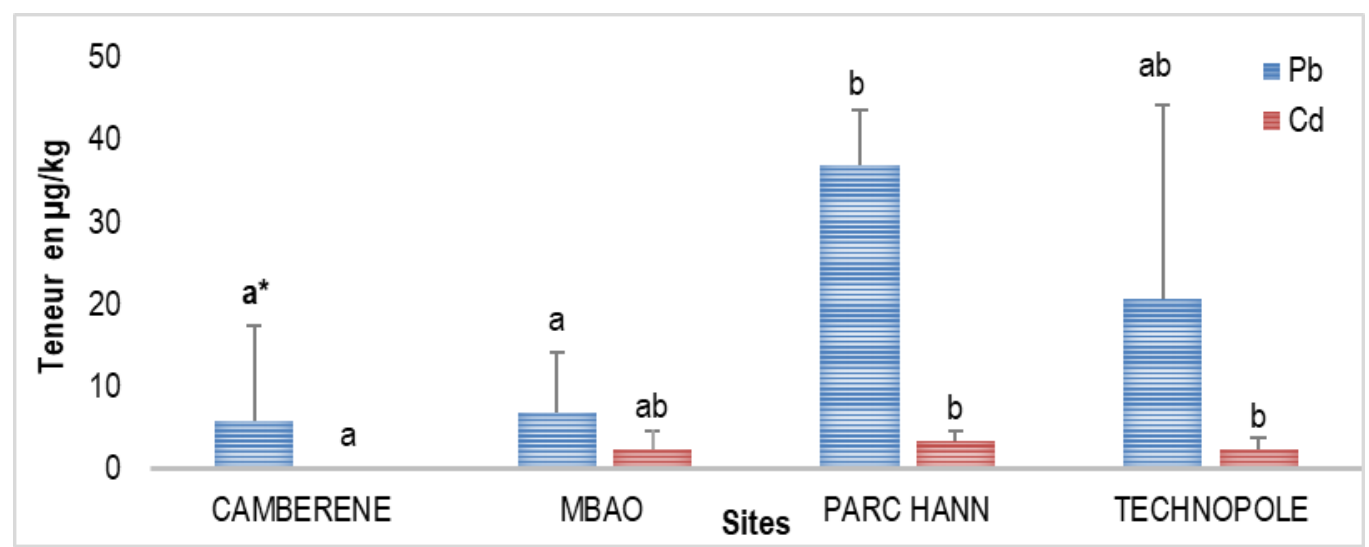

Figure 3: Distribution des teneurs en $\mathrm{Pb}, \mathrm{Cd}$ et Fe dans la chair du tilapia

*Les lettres communes partagées pour chaque site et pour chaque métal ne présentent pas de différence significative.

Bioconcentration des métaux traces dans les Tilapias: Les tilapias pêchés dans l'étang du Technopôle concentrent plus de $\mathrm{Pb}$ avec un FBC de 506. Ceux qui concentrent moins de Pb sont pêchés dans l'étang de Cambérène avec un $\mathrm{FBC}$ de 44. Le maximum de bioconcentration du $\mathrm{Cd}$ est obtenu avec les tilapias péchés à Cambérène pour un FBC de 65 . Les Tilapias pêchés à Mbao concentrent moins le $\mathrm{Cd}$ avec un plus faible FBC égale à 8 .
Risques sanitaires liés à la consommation du tilapia : Pour évaluer le risque pour la santé lié à la consommation des poissons étudiés, seules les teneurs maximales en métaux traces ( $\mu \mathrm{g} / \mathrm{kg}$ de poids frais) de la partie comestible (chair) du tilapia et non les teneurs moyennes, sont prises en compte. Les résultats de l'évaluation de l'exposition au $\mathrm{Pb}$ et au $\mathrm{Cd}$ liée à la consommation de tilapia de même que les quotients de danger (THQ) correspondants chez les adultes sont rapportés dans le tableau 3 .

Tableau 3: Dose journalière d'exposition et quotient de danger chez les adultes

\begin{tabular}{|c|c|c|c|c|c|c|}
\hline ETM & $\mathrm{CO}(\mathrm{kg} / \mathrm{j})$ & C (mg/kg) & DJA (mg/kg/j) & $P(\mathrm{~kg})$ & DJE (mg/kg/j) & THQ \\
\hline $\mathrm{Pb}$ & \multirow{3}{*}{0,043} & 0,05715 & $36.10^{-4}$ & \multirow{3}{*}{60} & $40,9.10^{-6}$ & 0,011 \\
\hline & & & & & & \\
\hline $\mathrm{Cd}$ & & 0,00552 & $2.10^{-4}$ & & $39,6.10^{-7}$ & 0,009 \\
\hline
\end{tabular}

Selon les scénarios d'exposition susmentionnés, le THQ total incluant Plomb et Cadmium est inférieur à 1

\section{DISCUSSION}

Qualité des eaux des différents sites: Les concentrations en métaux traces sont déterminées sur les échantillons d'eau brute acidifiée sans filtration préalable. Les concentrations moyennes de $\mathrm{Pb}$ les plus élevées $\left(1,34 \mu \mathrm{g} . \mathrm{L}^{-1}\right)$ demeurent faibles par rapport aux exigences relatives à la qualité des eaux pour les cours d'eau qui sont de $10 \mu \mathrm{g} \cdot \mathrm{L}^{-1}$ (OEaux, 1998). La comparaison des résultats de l'analyse des métaux traces dans l'eau aux critères de qualité a permis de déceler que seul le $\mathrm{Cd}$ à l'étang de Mbeubeuss présente des concentrations moyennes qui dépassent les critères de qualité de l'eau $\left(0,22 \mu \mathrm{g} . \mathrm{L}^{-1}\right)$. Les valeurs obtenues dans cette étude sont comprises dans
$(\mathrm{THQT}=0,02)$ ce qui indique un faible risque sanitaire lié à la consommation de ces poissons.

l'intervalle de valeurs trouvées par Diop et al (2014). Ces derniers ont évalué la contamination, la distribution et la spéciation des métaux traces dans la colonne d'eau des côtes dakaroises où le $\mathrm{Pb}$ total oscillait entre 0,047 et $3,87 \mu \mathrm{g} \cdot \mathrm{L}^{-1}$ et les teneurs en $\mathrm{Cd}$ étaient entre 0,06 et $0,43 \mu \mathrm{g} \cdot \mathrm{L}^{-1}$.

Contamination par les métaux des tilapias dans les différents sites: Notre étude a concerné au total 20 poissons de même taille dont cinq poissons par site. Seul l'étang de Mbeubeuss était épargné du fait de la rareté du poisson dans ce site au moment de nos échantillonnages. Dans tous les sites, les teneurs en métaux traces dans le foie étaient supérieures à celles 
retrouvées dans la chair à l'exception du Cd à Mbao, au Parc Hann et au site Technopôle comme préalablement décrits dans d'autres travaux (Henry et al, 2004 ; Diop et al, 2016). En effet, dans les organes à activité métabolique élevée comme le foie où la métallothionéine lie les métaux, l'accumulation d'éléments est plus importante que dans les organes à activité métabolique inférieure, tels que les muscles (Ploetz et al, 2007). Par ailleurs, des différences intersites dans les teneurs en métaux ont été observées (Figures 2 et 3 ). Les tilapias du Parc Hann sont significativement $(p<0,05)$ les plus contaminés en $\mathrm{Pb}\left(145 \mu \mathrm{g} \cdot \mathrm{kg}^{-1}\right.$ de poids frais) et les plus riches en fer

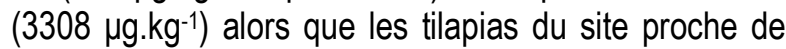
l'usine de traitement des eaux usées (Cambérène) sont significativement $(p<0,05)$ plus contaminés en $\mathrm{Cd}$. Les teneurs en $\mathrm{Pb}$ et en $\mathrm{Cd}$ trouvées dans la chair des Tilapias sont inférieures aux normes préconisées par I'Union Européenne (EC, $2014 ;$ 2015). Ces teneurs sont inférieures à celles trouvées par Youssao et al (2011).L'indice de pollution calculé qui considère tous les métaux et tous les poissons prélevés au niveau de chaque étang a permis de classer les sites du plus pollué au moins pollué: Cambérène > Parc Hann > Mbao > Technopôle suggérant que le site Technopôle est le moins pollué et que Cambérène et Parc Hann enregistrent les tilapias les plus contaminés. Le site Cambérène est proche de l'usine de traitement des eaux usées de Dakar. C'est aussi un site qui accueille toutes les eaux de ruissellement provenant du maraichage mais aussi un dépotoir d'ordure de tout genre. Le Parc Hann est le site où on ne rencontre ni de zone de maraichage, ni de dépôt d'ordure, ni d'activité pouvant directement être à l'origine d'une contamination de l'étang. $\mathrm{Le} \mathrm{Pb}$ et le $\mathrm{Cd}$ étaient en dessous de la limite de détection lors du dosage des échantillons d'eau provenant du site Parc Hann. Les

\section{CONCLUSION}

La présente étude a porté sur deux matrices des étangs que sont la colonne d'eau et le poisson tilapia. En ce qui concerne la colonne d'eau, seule la teneur moyenne en $\mathrm{Cd}$ au site Mbeubeuss dépasse les critères de qualité de l'eau. Pour les poissons, l'indice de pollution des métaux a révélé que Technopôle est le site le moins pollué alors que Cambérène et Parc Hann enregistrent les tilapias les plus contaminés. Les teneurs moyennes en $\mathrm{Pb}$ et en $\mathrm{Cd}$ trouvées dans la chair du tilapia sont inférieures aux normes préconisées par I'Union Européenne. L'évaluation des risques sanitaires a montré que les quotients de danger teneurs en $\mathrm{Pb}$ et en $\mathrm{Cd}$ retrouvées dans les poissons proviendraient du sédiment puisque le tilapia peut se comporter comme une espèce benthique. Par ailleurs, les FBC trouvés confirment que les tilapias pêchés à Cambérène, Mbao et Technopôle ont concentré les métaux à partir de l'eau dans laquelle ils vivent.

Risques sanitaires liés à la consommation de tilapia: L'accumulation des ETM dans les poissons Tilapia peut entrainer des effets néfastes pour la santé des populations humaines qui consomment régulièrement ces poissons. Pour évaluer le risque pour la santé associé à la consommation des poissons étudiés, la concentration moyenne de chaque contaminant a été comparée aux valeurs recommandées par l'UE pour la consommation humaine. Ainsi, tous les poissons étudiés avaient des teneurs en $\mathrm{Pb}$ et en $\mathrm{Cd}$ dans la chair inférieures aux limites maximales recommandées par l'Union Européenne pour la consommation humaine (EC, 2014 ; EC, 2015). Le quotient de danger (THQ), largement utilisé pour l'évaluation des risques potentiels pour la santé humaine liés à l'exposition aux produits chimiques a été calculé. Si le $T H Q>1$, il peut y avoir une préoccupation pour les effets potentiels sur la santé (USEPA, 2000). Nos résultats montrent que la survenue d'un effet toxique provenant du $\mathrm{Pb}(\mathrm{THQ}=$ $0,011<1)$ ou du $\mathrm{Cd}(\mathrm{THQ}=0,009<1)$ lié à la consommation des tilapias est peu probable (tableau 3). En effet, le calcul du THQ total incluant le $P b$ et le $\mathrm{Cd}$ est inférieur à $1(\mathrm{THQT}=0,02)$ et n'indique pas de danger lié à la consommation par la population locale de ces poissons. Diop et al (2017) ont obtenu des résultats similaires dans leur étude portant sur les risques sanitaires potentiels liés à la consommation de poissons contaminés par des métaux traces le long des côtes sénégalaises.

obtenus pour le $\mathrm{Pb}$ et le $\mathrm{Cd}$ sont inférieures à 1 ce qui suggère que la consommation des poissons étudiés ne présentent pas de risques importants pour la santé des consommateurs. Notre travail a permis de mettre en lumière le niveau de contamination en $\mathrm{Pb}, \mathrm{Cd}$ et $\mathrm{Fe}$ des plans d'eau et du poisson tilapia péché aux étangs de Dakar et les risques sanitaires associés afin de contribuer à la sensibilisation des autorités et des populations pour la préservation de ces zones humides en perspective de l'atteinte des objectifs 2, 6 et 14 de développement durable. 


\section{REFERENCES}

Amara R Diop M, Diop C, Ouddane B, 2019. The senegalese coastal and marine Environment. In book: World Seas: An Environmental Evaluation Volume I: Europe, The Americas and West Africa. Charles Sheppard, $2^{\text {nd }}$ Edition, Elsevier, Londre, Angleterre, Chapter 37: 855-873.

ANSD, 2009. Situation économique et sociale du Sénégal en 2008. République du Sénégal, Ministère de l'Economie, des Finances et du Plan. 14pp

ANSD, 2013. Recensement Général de la Population et de l'Habitat, de l'Agriculture et de l'Elevage. Rapport définitif, République du Sénégal, Ministère de l'Economie, des Finances et du Plan. p 19

Bandowe BAM, Bigalke M, Boamah L, Nyarko E, Saalia FK, Wilcke W, 2014. Polycyclic aromatic compounds (PAHs and oxygenated PAHs) and trace metals in fish species from Ghana (West Africa): bioaccumulation and health risk assessment. Environ Int. 65:135 - 146

Bisson M, Vivier S, La Rocca B et Gourland C, 2009. Point sur les Valeurs Toxicologiques de Référence (VTR), rapport d'étude 17/03/2009, $\mathrm{N}^{\circ}$ DRC-08-94380- 11776C, INERIS, [En ligne] URL : http://www.ineris.fr/hml, consulté le 28/10/2017 à $13 \mathrm{~h} 44 \mathrm{~min}$.

Casas S, 2005. Modélisation de la bioaccumulation de métaux traces ( $\mathrm{Hg}, \mathrm{Cd}, \mathrm{Pb}, \mathrm{Cu}$ et $\mathrm{Zn}$ ) chez la moule, Mytilus galloprovincialis, en milieu méditerranéen. Interactions entre organismes. Thèse Doctorat. Université du Sud Toulon Var, France, 364pp.

Diop C, Dewaelé D, Diop M, Touré A, Cabral M, Cazier F, Fall M, Diouf A, Ouddane B, 2014. Assessment of contamination, distribution and chemical speciation of trace metals in water column in the Dakar coast and the Saint Louis estuary from Senegal, West Africa. Marine Pollution Bulletin 86: 539 - 546.

Diop M, Howsam M, Diop C, Cazier F, Goossens JF, Diouf A, Amara R, 2016. Spatial and seasonal variations of trace elements concentrations in liver and muscle of round Sardinelle (Sardinella aurita) and Senegalese sole (Solea senegalensis) along the Senegalese coast. Chemosphere 144:758-766.

Diop M, Net S, Howsam M, Lencel P, Watier D, Grard T, Duflos G, Diouf A, Amara R, 2017. Concentrations and Potential Human Health Risks of Trace Metals ( $\mathrm{Cd}, \mathrm{Pb}, \mathrm{Hg}$ ) and Selected Organic Pollutants (PAHs, PCBs) in Fish and Seafood from the Senegalese Coast. Int J Environ Res. 11 (3) : 349 - 358.

EC, 2014. No 488/2014 of 12 May 2014 amending Regulation (EC) no 1881/2006 as regards the maximum levels for cadmium in foodstuffs. Off. J. Eur. 5. L 138/75

EC, 2015. No 2015/1005 of 25 June 2015 amending Regulation (EC) no 1881/2006 as regards the maximum levels for lead in foodstuffs. Off. J. Eur. 5. L 161/9.

FAO, 2014. La situation mondiale des pêches et de l'aquaculture. Possibilités et défis, Rome, Italie. 275pp.

FAO, 2016. Situation mondiale des pêches et de l'aquaculture. Contribuer à la sécurité alimentaire et à la nutrition de tous. Rome, Italie. 224pp.

Henry F, Amara R, Courcot L, Lacouture D, Bertho ML, 2004. Heavy metals in four fish species from the French coast of the Eastern English Channel and Southern Bight of the North Sea. Environ. Int. 30 (5): 675 - 683.

lqbal J, Shah MH, 2014. Study of seasonal variations and health risk assessment of heavy metals in Cyprinus carpio from Rawal Lake, Pakistan. Environ Monit Assess. 186: 2025 - 2037.

Khillare PS, Jyethi DS, Sarkar S, 2012. Health risk assessment of polycyclic aromatic hydrocarbons and heavy metals via dietary intake of vegetables grown in the vicinity of thermal power plants. Food Chem Toxicol 50: 1642 - 1652.

MDDELCC, 2014. Protocole d'échantillonnage de l'eau de surface pour l'analyse des métaux en traces. Direction du suivi de l'état de l'environnement, Québec, ISBN 978-2-55069205-8 (PDF), 19 pp.

OEaux, 1998. Ordonnance du 28 octobre 1998 sur la protection des Eaux. Suisse, Etat le 1er juin 2018. 
Ploetz M, Fitts BE, Rice TM, 2007. Differential accumulation of heavy metals in muscle and liver of a marine fish, (King Mackerel, Scomberomorus cavalla Cuvier) from the Northern Gulf of Mexico. USA. Bull. Environ. Contam. Toxicol. $78: 124$ - 127.

Storelli MM, 2008. Potential human health risks from metals $(\mathrm{Hg}, \mathrm{Cd}$, and $\mathrm{Pb})$ and polychlorinated biphenyls (PCBs) via seafood consumption: Estimation of target hazard quotients (THQs) and toxic equivalents (TEQs). Food and Chemical Toxicology 46: 2782 - 2788.

Taweel A, Shuhaimi-Othman M, Ahmad A, 2013. Assessment of heavy metals in tilapia fish (Oreochromis niloticus) from the Langat River and Engineering Lake in Bangi, Malaysia, and evaluation of the health risk from tilapia consumption. Ecotoxicol Environ Saf. 93: 45 51.

USEPA, 2000. Guidance for assessing chemical contaminant. Data for use in fish advisories. Fish sampling and analysis, 3rd edn. Washington DC: Office of Water. [EPA 823-R95-007].

Youssao A, Soclo HH, Bonou C, Vianou K, Gbaguidi M et Dovonon L, 2011. Evaluation de la contamination de la faune ichthyenne dans le complexe lagunaire Nokoué - chenal de Cotonou par le plomb : cas des espèces Sarotherodon melanotheron, Tilapia guineensis et Hemichromis fasciatus (Bénin). Int. J. Biol. Chem. Sci. 5(2): 595 - 602. 